\title{
Link travel time estimation based on large-scale low-frequency floating car data
}

\author{
Yuguang Li \\ State Key Laboratory of Information Engineering in \\ Surveying, Mapping and Remote Sensing \\ Wuhan University \\ Wuhan, China \\ wd_ygli@sina.com
}

\author{
Chaoyang Shi \\ State Key Laboratory of Information Engineering in \\ Surveying, Mapping and Remote Sensing \\ Wuhan University \\ Wuhan, China \\ chaoyangshi@whu.edu.cn
}

\author{
Qingquan Li \\ Shenzhen Key Laboratory of Spatial Spatial Smart Sensing and Services \\ Shenzhen University \\ Shenzhen, China \\ liqq@szu.edu.cn
}

\begin{abstract}
With advances in information technologies, floating car data (FCD) (e.g. trajectories of taxis) becomes an important data source for collecting traffic information in urban road networks. Due to the low sampling frequency of FCD, it is a challenge to obtain accurate and reliable travel time estimation based on FCD. This paper presents a location-speed method for accurately estimating travel times in signalized road networks based on FCD. The proposed model utilizes trajectories of probe vehicles on the upstream and downstream of signalized intersections in order to estimate delays caused by traffic signals. A large-scale case study using real-world FCD in Wuhan is carried to validate the proposed method. The results of case study show that the proposed method can obtain satisfied estimation of travel times in signalized road networks.
\end{abstract}

Index Terms - travel time information, floating car data, signalized road networks

\section{INTRODUCTION}

Accurate and reliable traffic information is critical for Intelligent Transportation System (ITS). Travel time estimation is not only one of the most important indicators to evaluate network performance and but also a key input for transportation planning [1]. With the development and widespread application of Global Positioning System (GPS) technique, mobile detection technology (e.g. floating car technology) has been regarded as a cost-effective technique for collecting traffic information, due to its short construction period, low cost and large spatial coverage. In addition, the floating car data (FCD) can also be very useful for investigating travelers' route choice behaviors.

In the literature, a considerable amount of research efforts have been given to the development of travel time estimation methods. A well-developed method is the velocity integral method [2]. This method utilizes numerical integration theory to calculate the distance that a probe vehicle traveled from collected discrete GPS sampling points. Based on FCD, a travel time estimation method was proposed by analyzing the dwell time of probe vehicles and link lengths [3], [4]. Researchers also utilized FCD to estimate other traffic parameters, such as mean travel speed, OD demands and validate the results using VISSIM simulation software [5]. The effects of different FCD sample sizes and sampling frequency on link travel time estimation were investigated [6], [7]. Trajectory reduction approach was presented to calculate the trajectory of floating car, and estimate average link travel speed according to trajectory time [8].

The above link travel time estimation algorithms only focused on FCD with a sampling frequency (i.e. less than 10s), and delays caused by traffic signals have not been considered. At present many Chinese megacities have established the taxi monitoring system, in which the sampling interval of collected GPS FCD are more than 30s, referred to as the low-frequency floating car data. This all-weather real-time traffic data on any part of large road networks are the important data source of urban road traffic information. The link travel time estimation method using conventional frequency GPS data can't be directly adopted because of their low sampling frequency, therefore, new methods to estimate urban link travel time should be proposed to deal with the low frequency characteristics of FCD. At present, some scholars have studied estimation methods of urban link travel time with FCD, including weighted speed-distance method [9], and road subsection analysis method [10]. These two methods realize estimating link travel time based on FCD, but are not suitable for traffic light control links that spread over the road networks in the city. In this paper, after analyzing a large number of real FCD on the roads near the intersections, a link travel time estimation method which takes into consideration the impact of intersection traffic lights based on the FCD (e.g. sampling 
interval is more than 40s) is proposed to improve the accuracy and reliability of link travel time estimation.

\section{EXISTING PROBLEMS}

\section{A. Link and link travel time}

Intersections are the key nodes of road traffic in the city, and there are traffic lights controling the vehicle stream. Roads can be represented by line segments, while the centers of intersections are line endpoints respectively in road navigation electronic map. In this paper, road between two traffic lights are divided into links when using the road navigation map data and FCD to estimate link travel time, and the time passing the link is link travel time.

\section{B. Limitations and of the current algorithms}

Basically, FCD are distributed on links near the intersection for the reason of long sampling interval and dynamically passing through road intersection as shown in Fig. 1. Hence, the time floating cars go through intersection is almost impossible to obtain directly and can only be obtained by estimating the instant vehicles pass through intersection with FCD of upstream and downstream, Linear Interpolation (LI) is the commonly used method.

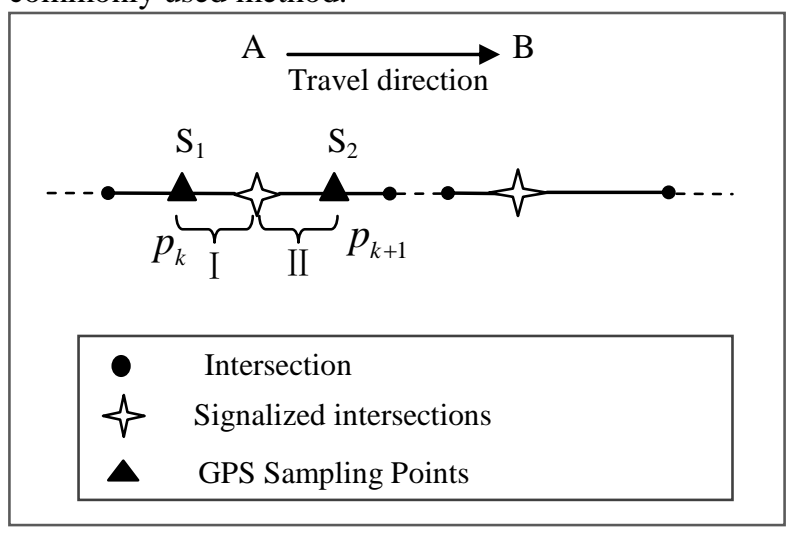

Fig. 1. The link situation of signalized intersections

In Fig. $1, S_{1}, S_{2}$ represent links, $A, B$ denote intersections, $p_{k 1}, p_{k+1}$ stand for the location of sampling points, and $T_{k}, T_{k+1}$ are sampling instant respectively. For process $I$, floating car is approaching intersection $A$ from link $S_{1}$ and $L_{I}$ is the travel distance. While floating car departs from intersection $A$ to link $S_{2}$ with a distance $L_{I I}$ in process $I I$ which includes the process through the intersection.

1) Linear Interpolation Method (LI)

Linear interpolation is based on relationship of the sampling time and intersection location of FCD to estimate the instant of going through intersection. The instant $T_{A}$ vehicles pass through intersection $A$ is calculated as follows

$$
T_{A}=T_{K}+\frac{L_{I}}{L_{I}+L_{I I}}\left(T_{K+1}-T_{K}\right)
$$

All FCD can be processed by this method, however, it assumes that the vehicles are traveling at a constant speed on the links
$S_{1}$ and $S_{2}$, which ignores the impact of the traffic light. Because of this reason, vehicles go slow or stop possibly, and the accuracy of $T_{A}$ is influenced. In addition, GPS signals can be affected by various factors in the big cities, which results in a longer sampling interval and a bigger error.

2) Weighted Speed-Distance Method

This method was proposed by Zhu (2008), which analyzed two situations of intersection link length. Without considering the length of road intersection, the average speed of floating cars on link 1 is calculated by

$$
V_{1}=\left(V_{s_{2}} \cdot L_{1}+V_{s_{1}} \cdot L_{2}\right) / V_{s_{2}} \cdot \Delta T
$$

Where $\Delta T$ is the difference of $T_{2}$ and $T_{1}$. Under the condition of considering the length of the road intersection, the average speed is calculated from the following formula

$$
V_{1}=\frac{L_{1}-0.5 \cdot L_{\text {Cross }}}{L_{1}} \cdot V_{s_{1}}+\frac{0.5 \cdot L_{\text {Cross }}}{L_{1}} \cdot V_{\text {Cross }}
$$

Where $V_{\text {Cross }}$ denotes the speed of floating car on intersection link, which is calculated by

$$
V_{\text {Cross }}=\frac{L_{\text {Cross }} \cdot V_{s_{1}} \cdot V_{s_{2}}}{V_{s_{1}} \cdot V_{s_{2}} \cdot \Delta T-V_{s_{2}} \cdot\left(L_{1}-0.5 \cdot L_{\text {Cross }}\right)-V_{s_{1}} \cdot\left(L_{2}-0.5 \cdot L_{\text {Cross }}\right)}
$$

According to Eq. 2, when $V_{s_{1}}$ is equal to zero, $V_{1}$ is updated to $\frac{L_{1}}{\Delta T}$, which is obviously unreasonable. However, most $V_{s_{1}}$ are zero value in actual intersections controlled by traffic signal, the speed $V_{\text {Cross }}$ in Eq. 4 tends to appear unreasonable situation, which leads to unreliable calculation result of Eq. 3.

\section{3) Road Subsection Analysis Method}

Yue et al. (2009) proposed this method, which artificially divided a link into two parts: Smooth Part (SP) which is far away from travel direction and Intersection Part (IP), and identified the distribution two consecutive GPS sampling data as six types based on their relationship with SP and IP. But when two sampling data span more than one intersection, Weighted Speed-Distance Method is adopted, which results in the same problem when estimating the travel time under the situation of traffic signal control.

Consequently, a new link travel time estimation method should be put forward to improve the accuracy and reliability of travel time estimation results, based on vehicle travel patterns on travel signal control links and the characteristics of FCD.

\section{LINK TRAVEL TIME ESTIMATION METHOD BASED ON FCD ON THE DOWNSTREAM LINK OF THE INTERSECTION}

\section{A. Travel characteristic analysis for vehicles on the links near signalized intersections}

The traffic flow is very big in Chinese megacities, roads are often congested. For the intersection with traffic signal control, the release time is a quarter of a cycle, while vehicles are in a state of stop or amble in other three-quarters, and need multiple signal cycles to enter the intersection. Vehicles accelerate first 
after through the intersection, and drive at a constant speed when reaching a certain speed, Fig. 2 shows this process.

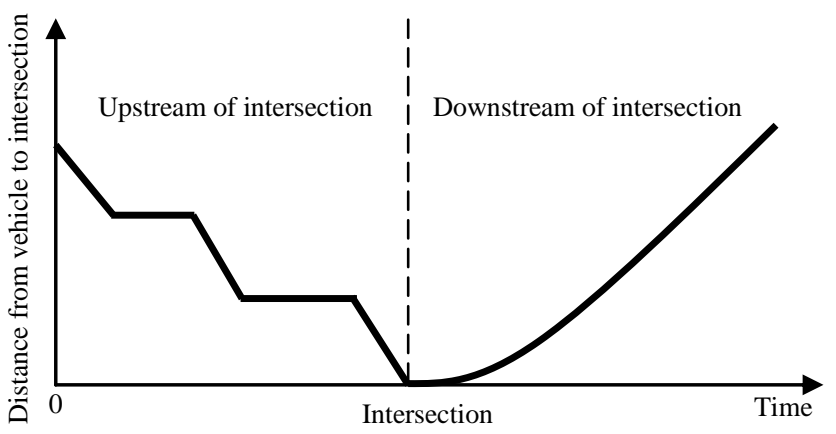

Fig. 2. The vehicle travel schematic diagram on the upstream and downstream links of signalized intersection

In Fig. 2, the horizontal axis means time, and the longitudinal axis represents the distance vehicles are from the intersection. As shown in Fig. 2, vehicle speed is very unstable before entering the intersection, so the vehicle instantaneous speed collected by low-frequency floating car don't represent the vehicle travel state on the upstream link of the intersection.

\section{B. Distribution of FCD on the upstream and downstream links of signalized intersections}

To estimate more accurate and reliable link travel time for urban road networks with signalized intersections, FCD on the links near signalized intersections are analyzed. As we can see from the previous discussion, the main factors influencing the calculation accuracy of the instant vehicles pass through intersection are the location of floating cars and instantaneous speed. In fact the instantaneous speeds of most floating cars on the upstream links closest to the intersection are equal to zero, and there is no pattern for other speed. However, the instantaneous speeds of most floating cars on the downstream links closest to the intersection are above zero, and are correlative with the distance from the intersection, that is, the farther the distance, the greater the speed. The traffic of the downstream links of the intersection is quite smooth, vehicle travel model is constructed through the relationship of locationspeed, and the instant vehicles pass through the intersection can be derivated to further estimate link travel time.

\section{Travel pattern model for vehicles on the downstream link of the intersection}

Usually, vehicles accelerate from the intersection, and keep accelerating for a period of time until reaching the limited speed. The sampling interval of FCD in Wuhan is 40s, in which period vehilcles are in a state of acceleration, therefore, the location-speed relationship in a short time after passing through the intersection can be represented by

$$
V^{2}=2 \cdot a \cdot L+V_{0}^{2}
$$

Where $V$ is the instantaneous speed of floating cars at the sampling point after passing through the intersection; $a$ is the average acceleration between the sampling point and intersection; $L$ is the travel distance from the sampling point to the intersection; $V_{0}$ is the speed passing through the intersection.

The speed vehicles pass through the intersection can be approximately regarded as a constant due to its smallness. Therefore, in order to express conveniently, Eq. 5 is updated with the following equation

$$
V^{2}=p \cdot L+C
$$

$V$ and $L$ are obtained from FCD and matching results of FCD. When a floating car on the downstream link of the intersection has more than two sampling points in a certain period of time, the parameters $p$ and $C$ can be fitted with Least Square Method. The location-speed distribution of FCD in Wuhan within an hour on the downstream link of an intersection and the fitted vehicle motion curve are shown in Fig. 3 .

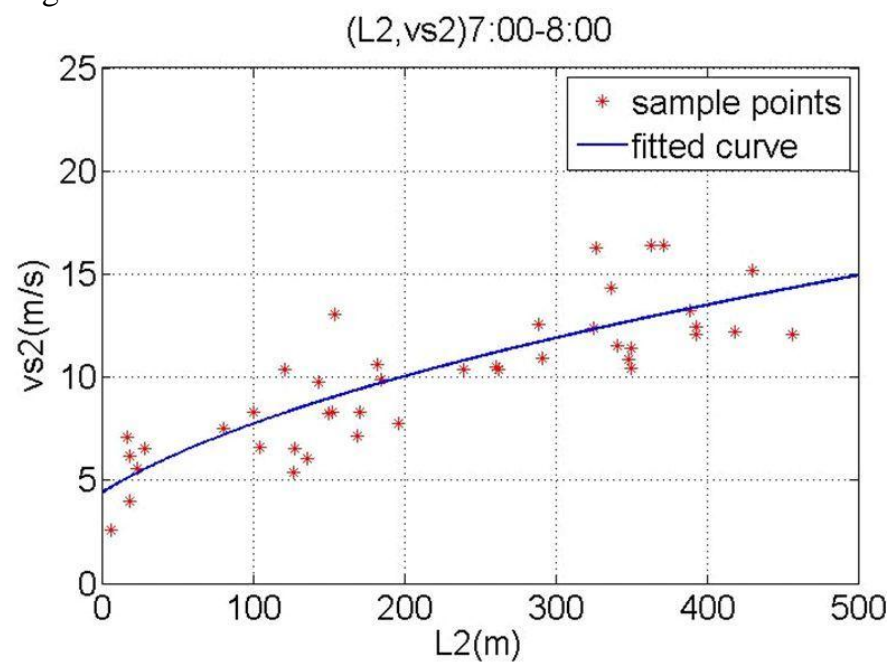

Fig. 3. The location-speed distribution of FCD within an hour on the downstream link and the fitted vehicle motion curve

\section{Link travel time estimation based on FCD on the downstream link of the intersection}

In a short period of time, road traffic flow is often quite stable, so the fitted vehicle motion curve based on multiple FCD is also reliable. The instants each vehicle passes through the intersection on the downstream link are calculated based on Eq. 5a as followings:

Step 1: Count the instantaneous speed $V$ and the distance $L$ to the intersection of all $N$ FCD on the downstream link of the intersection in a certain period of time.

Step 2: Fit $V$ and $L$ with Least Square Method based on Eq. 5a, and obtain the parameters $p$ and $C$.

Step 3: Use the Intergral method to calculate the travel time $t$ of floating cars on the downstream link of the intersection

$$
t=\int_{0}^{L} \frac{1}{V} d L=\frac{2}{p} \cdot \sqrt{p \cdot L+C}-\sqrt{C}
$$

Step 4: $T_{I}$ represents the instant floating cars pass through the intersection $I$ as shown in Fig. 2,

$$
T_{I}=T_{k+1}-t
$$


Where $T_{k+1}$ is the instant of sampling point $p_{k+1}$.

Step 5: Similarly, calculate the instant $T_{I I}$ floating cars pass through the intersection $I I$

$$
T_{I I}=T_{k+1}^{\prime}-t^{\prime}
$$

Step 6: The travel time $T_{s}$ floating cars pass through the links between intersection $I$ and $I I$ is calculated by

$$
T_{s}=T_{I I}-T_{I}
$$

\section{CASE STUDY}

In order to validate the effectiveness of the algorithm, the real FCD of a typical weekday on 9 March 2009 (Monday) in Wuhan China are adopted in this article. The sampling interval is about 40 seconds, but also longer for some data. A major road (or path) from 'Luoyu Road' (intersection A) to 'Bayi Road' (intersection B) was selected as the study link, both of which were controlled by traffic lights.

Due to most travel speeds of FCD on the upstream link of the intersection are equal to zero as shown in Fig. 4, the travel speed and the instant calculated by Weighted Speed-Distance Method are completely wrong, nearly half of the calculated results appear negative as shown in Fig. 5, which is unreasonable.

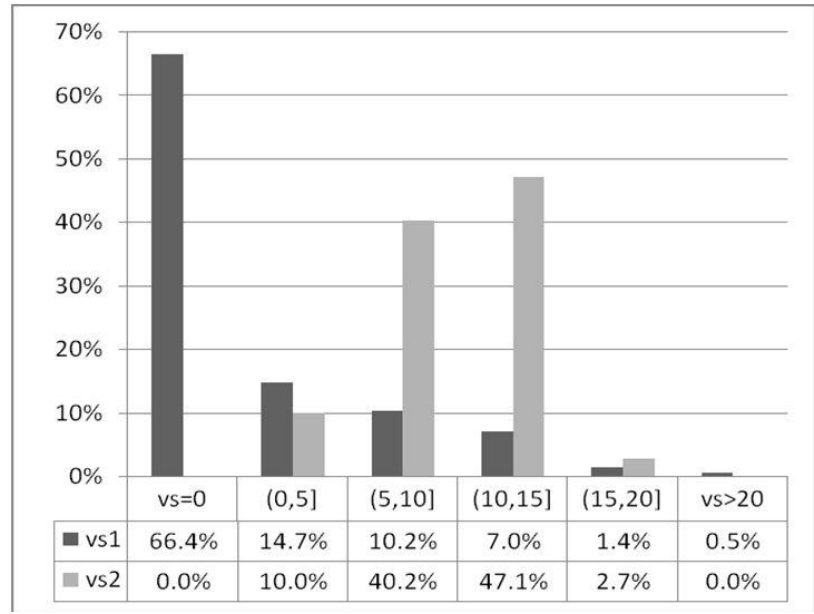

Fig. 4. The instantaneous speed distribution on the upstream and downstream links of signalized intersection

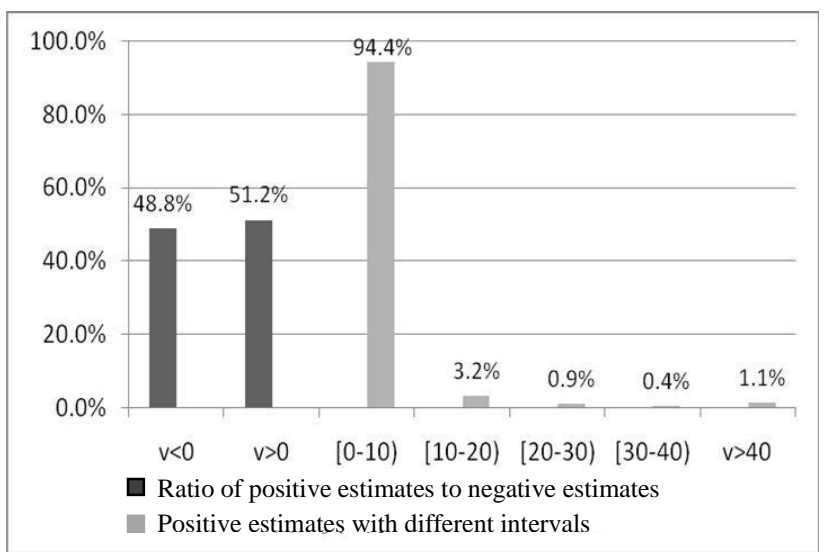

Fig. 5. The calculated results of link speed at the intersection
Therefore, Weighted Speed-Distance Method and Road Subsection Analysis Method are not used to carry out experimental computations in this paper, only linear interpolation algorithm is adopted to compare with the proposed algorithm. Link travel times are estimated at one hour interval from the morning peak to evening peak (07:00-22:00), the results of two algorithms are shown in Table I.

TABLE I. THE RESULTS OF TWO TRAVEL TIME ESTIMATION ALGORITHMS

\begin{tabular}{cccccc}
\hline \hline $\begin{array}{c}\text { Time } \\
\text { interval }\end{array}$ & $\begin{array}{c}\text { T(Proposed } \\
\text { method) }\end{array}$ & $\begin{array}{c}T 1 \\
(\mathbf{L I})\end{array}$ & dt_A & dt_B & $d T$ \\
\hline 7:00-8:00 & 126 & 154 & 43 & 15 & 28 \\
8:00-9:00 & 158 & 197 & 60 & 21 & 39 \\
9:00-10:00 & 119 & 162 & 60 & 16 & 43 \\
10:00-11:00 & 112 & 150 & 48 & 10 & 38 \\
11:00-12:00 & 99 & 132 & 40 & 7 & 33 \\
12:00-13:00 & 104 & 139 & 38 & 2 & 35 \\
13:00-14:00 & 114 & 146 & 45 & 13 & 32 \\
14:00-15:00 & 110 & 165 & 56 & 1 & 55 \\
15:00-16:00 & 115 & 147 & 46 & 14 & 32 \\
16:00-17:00 & 129 & 135 & 30 & 24 & 6 \\
17:00-18:00 & 124 & 150 & 35 & 9 & 26 \\
18:00-19:00 & 135 & 145 & 28 & 18 & 10 \\
19:00-20:00 & 137 & 163 & 45 & 19 & 26 \\
20:00-21:00 & 134 & 165 & 47 & 16 & 31 \\
21:00-22:00 & 122 & 157 & 53 & 18 & 35 \\
\hline \hline
\end{tabular}

In Table I, dt_A and dt_B are respectively the time delays of the estimated instant using the proposed method compared with LI algorithm due to the effects of traffic light when passing through the intersection A and B. $d T$ is the difference of $T 1$ and $T$.

As we can see from Table I, the results of LI algorithm are significantly delayed compared with that of the proposed method, which is because the average speed LI adopts is smaller than the real travel speed of the downstream link, this proves the rationality of the proposed algorithm. The reason that time delays are longer at intersection $\mathrm{A}$ is that the sampling interval is considerably larger than 40s, even up to 80-100s. There are systemic differences between the proposed algorithm and LI algorithm, however, the estimated results of the proposed algorithm are more consistent with the actual situation.

\section{CONCLUSIONS}

This paper presented a location-speed method for estimating travel times in signalized road networks based on large-scale low frequency floating car data (FCD). Delays caused by traffic signals were derived through the analysis of vehicle driving patterns on the upstream and downstream of the signalized intersection. A case study using real-world FCD in Wuhan was carried out to demonstrate the applicability of the proposed method. Numerical results demonstrated that the proposed algorithm has significant advantage over existing 
linear interpolation algorithm for esitmating delays caused by traffic signals.

\section{ACKNOWLEDGMENT}

The work described in this paper was jointly supported by grants from Shenzhen Scientific Research and Development Funding Program (No. ZDSY20121019111146499), and Shenzhen Dedicated Funding of Strategic Emerging Industry Development Program (No. JCYJ20121019111128765).

\section{REFERENCES}

[1] Y. Chen, L. Gao, Z. Li and Y. Liu, "A new method for urban traffic state estimation based on vehicle tracking algorithm", in IEEE Intelligent Transportation Systems Conference: pp.10971101, IEEE, Seattle, WA, USA, 2007.

[2] B. S. Kerner, C. Demir, R. Herrtwich, S. L Klenov, H. Rehborn, M. Aleksi and A. Haug, "Traffic state detection with floating car data in road networks", in 8th International IEEE Conference on Intelligent Transportation Systems, pp. 44-49, IEEE, Vienna, Austria, 2005.

[3] C. A. Quiroga and Bullock D. "Travel time studies with global positioning and geographic information systems: an integrated methodology". Transp. Res. Pt. C-Emerg. Technol., Vol.6, pp.101-127, 1998.
[4] J. Weng, J. Rong, Q. Yu and F. Ren, "Optimization on Estimation Algorithms of Travel Speed Based on the Real-time Floating Car Data", J. Beijing Univ. Technol., Vol.33 (5), pp.459-464, 2007.

[5] Y. Yue, H. Zou and Q. Li 2009. "Urban Road Travel Speed Estimation Based on Low Sampling Floating Car Data", ICCTP 2009, DOI 10.1061/41064(358)242, pp.1-7.

[6] Z. Yang, "Study on the Synthetic Link Travel Time Prediction Model of Key Theory of ITS", J. Traffic Transp. Eng., Vol.1 (1), pp.65-67, 89, 2001.

[7] C. Zhang, X. Yang and X. Yan, "Traffic Data Collection System Based on Floating Cars", Comput. Commun., Vol. 24(5), pp.3134, 2006.

[8] H. Zhang, Y. Zhang and H. Wen, "Estimation approaches of average link travel time using GPS data", J. Jilin Univ. (Eng. Technol. Edition), Vol.37 (3), pp.533-537, 2007.

[9] G. Jiang, A. Chang and W. Zhang, "Comparison of link travel2time estimation methods based on GPS equipped floating car", J. Jilin Univ. (Eng. Technol. Edition), Vol.39 (Sup.2), pp.182-186, 2009.

[10] L. Zhu and D. Yang, "Dynamic Travel Speed Collection Technology based on Low Frequency FCD", J. Transp. Syst. Eng. and Inf. Technol.,Vol.8(4), pp.42-48, 2008. 